\title{
Perceptual Global Illumination Cancellation in Complex Projection Environments
}

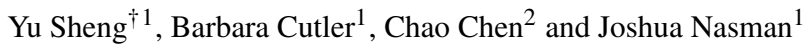 \\ ${ }^{1}$ Department of Computer Science, Rensselaer Polytechnic Institute, ${ }^{2}$ Institute of Science and Technology, Austria
}

\begin{abstract}
The unintentional scattering of light between neighboring surfaces in complex projection environments increases the brightness and decreases the contrast, disrupting the appearance of the desired imagery. To achieve satisfactory projection results, the inverse problem of global illumination must be solved to cancel this secondary scattering. In this paper, we propose a global illumination cancellation method that minimizes the perceptual difference between the desired imagery and the actual total illumination in the resulting physical environment. Using Gauss-Newton and active set methods, we design a fast solver for the bound constrained nonlinear least squares problem raised by the perceptual error metrics. Our solver is further accelerated with a CUDA implementation and multi-resolution method to achieve 1-2 fps for problems with approximately 3000 variables. We demonstrate the global illumination cancellation algorithm with our multi-projector system. Results show that our method preserves the color fidelity of the desired imagery significantly better than previous methods.
\end{abstract}

Categories and Subject Descriptors (according to ACM CCS): I.3.7 [Computer Graphics]: Three-Dimensional Graphics and Realism-Virtual reality, Radiosity

\section{Introduction}

As the brightness, contrast, resolution, and affordability of projectors increases, large-scale displays and projection systems are becoming more prevalent and can be integrated into our physical surroundings. Projection environments with multiple projection surfaces facing each other can suffer dramatically from the secondary scattering of light. This unintended reflection of light can lead to a scene with increased brightness, decreased contrast, and loss of color fidelity. To generate projection results that most faithfully capture the desired appearance, we must compute appropriate projection images that take into account this unintentional light scattering. Essentially we must solve an inverse global illumination optimization problem - given a physical scene and a desired appearance, we compute the optimal projected illumination such that the actual total illumination, which is the sum of projected illumination and secondary scattering, most closely matches the desired appearance.

For the examples in this paper, we transform the appearance of an existing physical geometry with diffuse white or

\footnotetext{
$\dagger$ shengyu@ cs.rpi.edu
}

light grey surfaces into colorful scenes. By modeling the diffuse illumination transport in the physical model, we are able to cancel indirect scattering of the projected light and more accurately control the total illumination of patches in the physical model. We address this problem using a patchbased radiosity framework [GTGB84]. We define $\mathbf{K}_{\mathbf{p}}$ to be the radiosity matrix for the physical model, $B_{d, i}$ to be the desired radiosity of each patch, and $B_{p, i}$ to be the radiosity (reflection of both direct and indirect illumination) of patch $i$ in the physical environment. $E_{p, i}$ is the necessary emitted light of patch $i$ in the physical scene, which will be provided by direct illumination from the projectors. Using the classic radiosity equation, reverse radiosity [ $\left.\mathrm{BGZ}^{*} 06, \mathrm{MKO} 06\right]$ assumes $B_{d, i}$ is always achievable, and lets $\mathbf{B}_{\mathbf{p}}=\mathbf{B}_{\mathbf{d}}$ :

$$
\mathbf{E}_{\mathbf{p}}=\mathbf{K}_{\mathbf{p}} \mathbf{B}_{\mathbf{p}}=\mathbf{K}_{\mathbf{p}} \mathbf{B}_{\mathbf{d}}
$$

Although this method provides an exact solution to Equation (1) with realtime computing rates, it may require negative emittances at some physical patches. To alleviate this issue, Sheng et al. [SYC10] proposed a bound constrained optimization method to find the optimal $\mathbf{E}_{\mathbf{p}}$ that minimizes the difference between $\mathbf{B}_{\mathbf{d}}$ and $\mathbf{B}_{\mathbf{p}}=\mathbf{K}_{\mathbf{p}}{ }^{-1} \mathbf{E}_{\mathbf{p}}$ in the linear $\mathrm{YPbPr}$ color space. However, the linear error metrics do not 
Y. Sheng, B. Cutler, C. Chen \& J. Nasman / Perceptual Global Illumination Cancellation in Complex Projection Environments

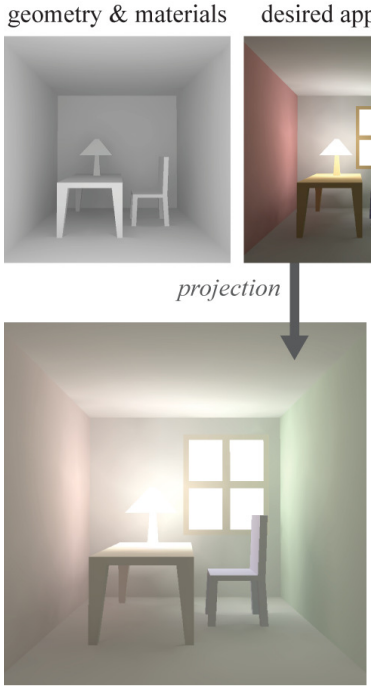

uncompensated exact radiosity inverse (reverse radiosity)

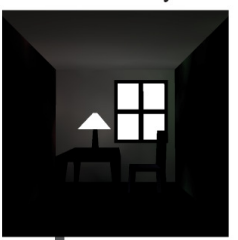

projection

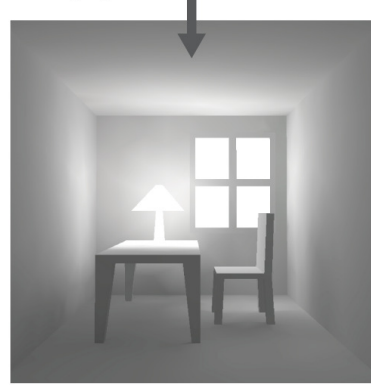

Bimber et al. 2006

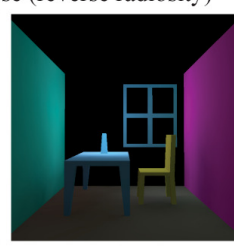

YPrPb optimization

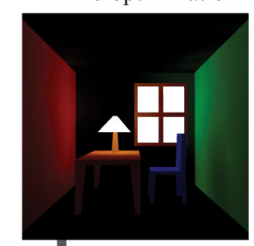

projection

Figure 1: We transform a simple diffuse white room into a more colorful room with simulated light emitted from the lamp shade and false windows. Simply projecting the desired imagery into the scene results in a washed out appearance due to indirect scattering. The exact inverse solution requires negative light that is discarded before projection. A linear optimization using YPbPr space produces a reasonable result, but we can guarantee the optimal result for human perception only by solving the problem in a perceptually uniform space, e.g., $L^{*} a * b *$.

match human perception, and sometimes produce noticeably different results (Figure 1).

We propose a perceptual global illumination cancellation method to faithfully preserve the contrast and color fidelity in multi-surface projection environments, such as the Office of the Future [RWC ${ }^{*} 98$ ] and architectural daylighting visualization [SYYC11]. Our contributions in this paper include:

- A formulation of the objective function in a perceptually uniform color space. We define the error metric in the CIE $\mathrm{L}^{*} \mathrm{a}^{*} \mathrm{~b}^{*}$ [McL76] color space, which is commonly used for measuring human perception of color difference.

- A fast nonlinear optimization algorithm to solve the perceptual objective function. We present a fast solver for the bound constrained nonlinear least squares problem with the Gauss-Newton and active set methods.

- We leverage GPGPU techniques and a multi-resolution strategy to further accelerate our nonlinear solver. Our algorithm achieves fast computing rates and can be used in iterative design applications.

\section{Related Work}

\subsection{Visual Difference Metrics}

The linear error metric proposed in Sheng et al.'s work [SYC10] is not a perceptual measurement of the difference. MacAdam ellipses [Mac42] demonstrate that equal distances in linear color spaces, such as RGB, YPbPr, or $\mathrm{XYZ}$, do not correspond to equal perceptual difference. In contrast, the CIE L*a* $b^{*}$ [McL76] color space was designed to be perceptually uniform. An RGB color triplet can be converted to a triplet $(L, a, b)$ in $\mathrm{L}^{*} \mathrm{a}^{*} \mathrm{~b}^{*}$ color space:

$$
\left[\begin{array}{l}
L \\
a \\
b
\end{array}\right]=\left[\begin{array}{c}
116 h\left(\frac{Y}{Y_{n}}\right)-16 \\
500\left(h\left(\frac{X}{X_{n}}\right)-h\left(\frac{Y}{Y_{n}}\right)\right) \\
200\left(h\left(\frac{Y}{Y_{n}}\right)-h\left(\frac{Z}{Z_{n}}\right)\right)
\end{array}\right],\left[\begin{array}{c}
X \\
Y \\
Z
\end{array}\right]=T\left[\begin{array}{c}
R \\
G \\
B
\end{array}\right],
$$

where $T$ is the $3 \times 3$ transformation matrix. $X_{n}, Y_{n}$, and $Z_{n}$ are CIE XYZ values of the reference white point. Function $h$ is a piecewise function defined by

$$
h(t)= \begin{cases}t^{\frac{1}{3}} & t>(6 / 29)^{3} \\ \frac{1}{3}\left(\frac{29}{6}\right)^{2} t+\frac{4}{29} & \text { Otherwise }\end{cases}
$$

The difference between any two colors in $\mathrm{L}^{*} \mathrm{a}^{*} \mathrm{~b}^{*}$ is defined as $\Delta E=\sqrt{\left(L_{1}-L_{2}\right)^{2}+\left(a_{1}-a_{2}\right)^{2}+\left(b_{1}-b_{2}\right)^{2}}$. Two colors are considered distinguishable if $\Delta E$ is greater than 2.3 [McL76]. Metrics based on the $\mathrm{L}^{*} \mathrm{a}^{*} \mathrm{~b}^{*}$ color space have been designed to evaluate the perceptual differences for complex color images [ZW97, CL07]; however, these error metrics are designed for 2D images and cannot be directly applied to our 3D global illumination cancellation problem.

\subsection{Inverse Lighting}

Projectors have been widely used to create immersive or semi-immersive environments for scientific visualization, education, and entertainment. Photometric calibration [MS04] and radiometric compensation [NPGB03, GB08] allow seamless display via multiple projectors on everyday surfaces. When projecting imagery into complex environments, effects such as reflection and refraction can be significant and should not be ignored.

Seitz et al. propose the inter-reflection cancellation operator (IRC), which, in theory, can be used to cancel indirect 
scattering for general BRDFs [SMK05]. However, acquiring the full IRC matrix is time consuming and requires significant storage. The light transport matrix $\left[\mathrm{SCG}^{*} 05\right]$ models full light transport between a camera image and projection image and captures a variety of global illumination phenomena. Methods based on the matrix [WB07, BCNR10] produce projection images by solving a linear equation. However, calibration of the transport matrix is both time and storage consuming, and furthermore, when the projection surfaces or projector configurations are modified the system must be fully re-calibrated. O'Toole and Kutulakos [OK10] propose a novel method to perform optical computing without explicitly capturing the matrix. For diffuse projection environments, reverse radiosity [BGZ*06, MKO06] and an optimization based cancellation method [SYC10] have been demonstrated to compensate for secondary scattering.

\subsection{Nonlinear Least Squares}

Many problems in computer vision and computer graphics can be posed as a Nonlinear Least Squares (NLSQ) optimization problem in the form

$$
\min _{\mathbf{x} \in \mathbf{R}^{n}}\|\mathbf{f}(\mathbf{x})-\mathbf{y}\|_{2},
$$

where $\mathbf{f}(\mathbf{x})$ is a smooth $\mathbf{R}^{n} \rightarrow \mathbf{R}^{m}(m \geq n)$ nonlinear function, $\mathbf{y} \in \mathbf{R}^{m}$. For the rest of the paper, we only assume $m=n$. For NLSQ problems, the second-order derivative (Hessian) can be well approximated by the first-order derivative (Jacobian). Gauss-Newton takes advantage of this fact and transforms the nonlinear problem to a linear least squares problem at each iteration. The Gauss-Newton method may not find a descent direction when the Jacobian matrix $\mathbf{J}$ of the objective function is rank deficient or close to rank deficient. The Levenberg-Marquardt (LM) method [Lev44] addresses this problem by adding a damping factor to the diagonal entries of matrix $\mathbf{J}^{\top} \mathbf{J}$. LM is a hybrid method of steepest descent and Gauss-Newton, and its convergence rate is often not as fast as Gauss-Newton.

Determining if a variable satisfies a bound constraint is straightforward; therefore, most bound constrained optimization solvers adopt the active set strategy. The active set method divides the variables into two sets, active and free variables. The active variables either satisfy the KKT condition or are close to bound constraints and can be solved by steepest descent. For free variables, traditional methods such as the Newton or Quasi-Newton may be used to compute a descent direction. Line search is then used to compute a step size, and the final result is projected to the feasible region.

\section{Perceptual Global Illumination Cancellation}

To optimize the final projection appearance for human perception, we formulate our objective function by measuring the difference between $\mathbf{B}_{\mathbf{p}}$ and $\mathbf{B}_{\mathbf{d}}$ in the CIE $\mathrm{L}^{*} \mathrm{a}^{*} \mathrm{~b}^{*}$ color space and enforcing the smoothness of $\mathbf{B}_{\mathbf{p}}$. Visual inspection and quantitative comparison show that our error metric preserves color fidelity significantly better than previous methods.

\subsection{Perceptual Error Metrics}

We define $L_{i}^{\prime}, a_{i}^{\prime}, b_{i}^{\prime}$ as the $\mathrm{L}^{*}, \mathrm{a}^{*}$, and $\mathrm{b}^{*}$ components of each patch for the desired appearance, and $L_{i}, a_{i}$ and $b_{i}$ as the resulting physical scene color with projection. $\mathbf{v}$ and $\mathbf{v}_{\mathbf{0}}$ are $3 n \times 1$ ( $n$ is the number of patches) vectors defined by:

$$
\begin{aligned}
\mathbf{v} & =\left[L_{1}, \ldots, L_{n}, a_{1}, \ldots, a_{n}, b_{1}, \ldots, b_{n}\right]^{\top}=[\mathbf{L}, \mathbf{a}, \mathbf{b}]^{\top} \\
\mathbf{v}_{\mathbf{0}} & =\left[L_{1}^{\prime}, \ldots, L_{n}^{\prime}, a_{1}^{\prime}, \ldots, a_{n}^{\prime}, b_{1}^{\prime}, \ldots, b_{n}^{\prime}\right]^{\top},
\end{aligned}
$$

$\mathbf{r}=\mathbf{v}-\mathbf{v}_{\mathbf{0}}$ is the difference vector.

The objective function consists of two parts, the absolute term and the spatial term.

Absolute Error Term. The first term defines the area weighted sum of absolute luminance and chrominance errors of the physical scene over all patches:

$$
\begin{aligned}
\phi_{a b s} & =\frac{\sum_{i} A_{i}\left[\left(L_{i}-L_{i}^{\prime}\right)^{2}+\left(a_{i}-a_{i}^{\prime}\right)^{2}+\left(b_{i}-b_{i}^{\prime}\right)^{2}\right]}{A_{\text {avg }}} \\
& =\mathbf{r}^{\top} \mathbf{W}_{\mathbf{1}} \mathbf{r},
\end{aligned}
$$

where $A_{i}$ is the area of patch $i$ in the physical scene, and is used to weight the importance of each patch based on its size. We normalize this term by dividing by the average area of all the patches. $\mathbf{W}_{\mathbf{1}}$ in Equation (5) is a $3 n \times 3 n$ diagonal matrix, with $\frac{A_{i}}{A_{\text {avg }}}$ as each diagonal element.

Spatial Error Term. Our second term aims to preserve the gradients and discontinuities between neighboring patches:

$$
\begin{aligned}
\phi_{s p t}= & \sum_{(i, j) \in n b d}\left[\left(L_{i}-L_{j}\right)-\left(L_{i}^{\prime}-L_{j}^{\prime}\right)\right]^{2}+\left[\left(a_{i}-a_{j}\right)-\left(a_{i}^{\prime}-a_{j}^{\prime}\right)\right]^{2} \\
& +\left[\left(b_{i}-b_{j}\right)-\left(b_{i}^{\prime}-b_{j}^{\prime}\right)\right]^{2}=\mathbf{r}^{\top} \mathbf{W}_{\mathbf{2}} \mathbf{r},
\end{aligned}
$$

where $(i, j) \in n b d$ indicates patches $i$ and $j$ share a common edge in the mesh. $\mathbf{W}_{\mathbf{2}}$ is a $3 n \times 3 n$ block diagonal matrix, whose diagonal element is the Laplacian matrix of the dual graph of the geometry.

Complete Objective Function. The complete objective function then can be computed as a weighted sum of $\phi_{a b s}$ and $\phi_{\text {spt }}$ :

$$
\begin{aligned}
\phi & =\alpha \phi_{a b s}+(1-\alpha) \phi_{s p t} \\
& =\mathbf{r}^{\top}\left[\alpha \mathbf{W}_{\mathbf{1}}+(1-\alpha) \mathbf{W}_{\mathbf{2}}\right] \mathbf{r}=\mathbf{r}^{\top} \mathbf{W r} .
\end{aligned}
$$

The equation can be denoted as a function of $\mathbf{E}_{\mathbf{p}}$ by further transforming $\mathbf{r}=\mathbf{v}-\mathbf{v}_{\mathbf{0}}$. For each triplet $\left(L_{i}, a_{i}, b_{i}\right)$ in $\mathbf{v}$, it can be rewritten as a nonlinear function of a triplet $\left(B_{r i}, B_{g i}, B_{b i}\right)$ in $\mathbf{B}_{\mathbf{p}}$, by Equation (2) and (3). Then the emittance $\mathbf{E}_{\mathbf{p}}$ can be computed from $\mathbf{B}_{\mathbf{p}}$ by equation (1).

We use the black level and maximum brightness of the projector as bound constraints for our optimization problem. The objective function $\phi$ can be written in the general form of bound constrained nonlinear least squares,

$$
\min _{\mathbf{x} \in \mathbf{R}^{\mathbf{n}}}\|\mathbf{f}(\mathbf{x})-\mathbf{y}\|_{2}, \text { such that } \mathbf{l} \leq \mathbf{x} \leq \mathbf{u},
$$



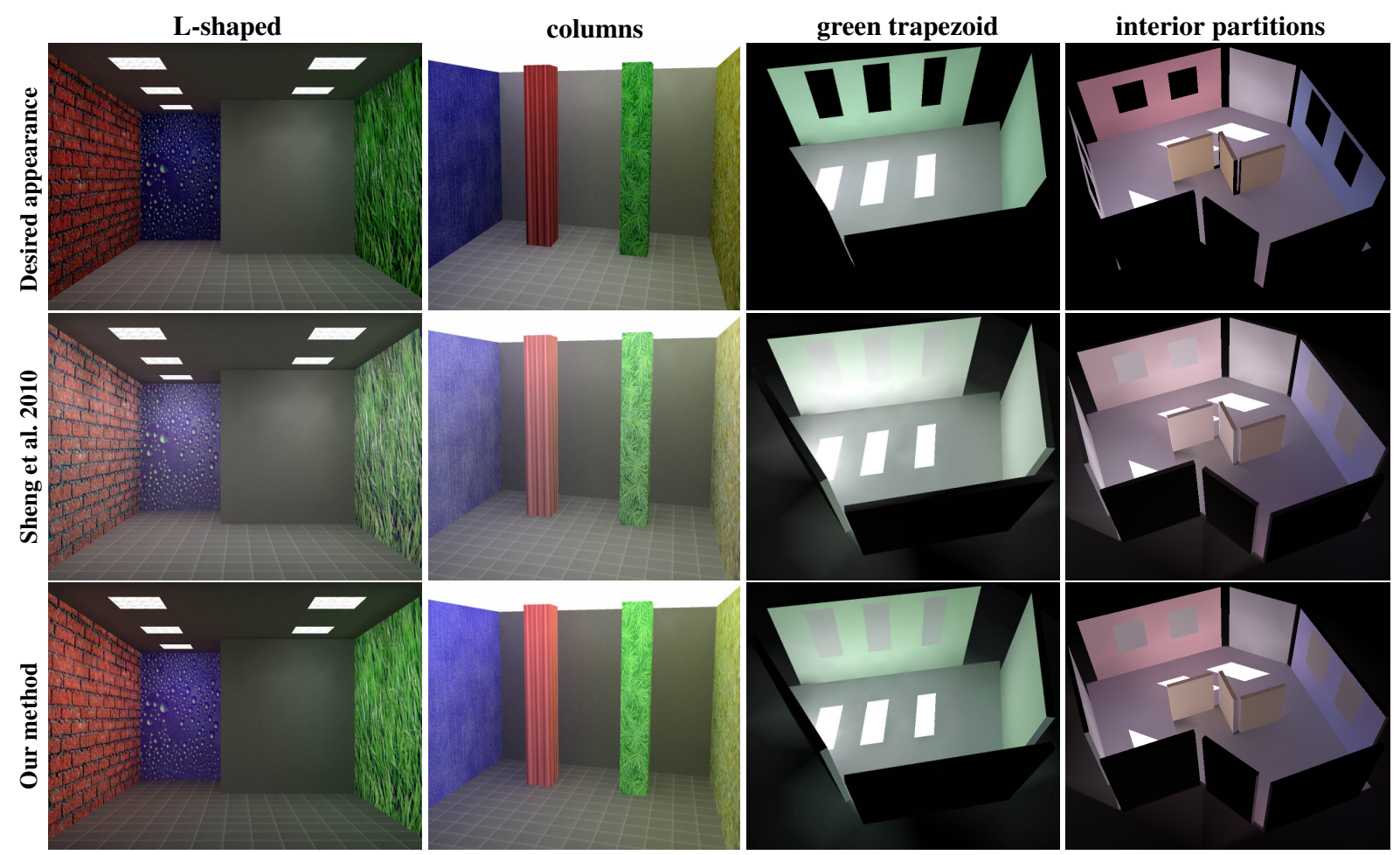

Figure 2: Four example scenes illustrate the differences between our perceptual compensation method and previous work. In each example we aim to transform geometry with diffuse white surfaces into the desired appearance shown in the top row. The first two examples demonstrate that high frequency detail textures may be combined with the per patch radiosity values.

where $\mathbf{f}(\mathbf{x})$ is a $\mathbf{R}^{\mathbf{n}} \rightarrow \mathbf{R}^{\mathbf{n}}$ nonlinear function, $\mathbf{y} \in \mathbf{R}^{\mathbf{n}}$. In section 4 , we propose an efficient solver for this problem.

\subsection{Comparison of Cancellation Algorithms}

We compare perceptual cancellation algorithm to Sheng et al.'s method [SYC10]. Figure 2 shows the results of several moderately complex comparison experiments. With these four examples, we observe that our method more faithfully preserves the saturated colors and contrast in the desired image than previous work. For each of the simulation images, the color difference between the desired appearance and the simulated projection results of both algorithms is computed using the CIE $\Delta E$ calculation. Table 1 lists the per-pixel average and maximum $\Delta E$ for each scene. In all of the examples, the perceptual cancellation algorithm produces much smaller numerical differences than Sheng et al.'s method.

\section{Efficient Nonlinear Solver}

Due to the nonlinearity of perceptual color space, we need to solve a nonlinear least squares problem defined by equation (6) with bound constraints. In this section, we prove two important properties of the objective function, and propose a fast nonlinear least squares solver based on the GaussNewton and active set methods.

\subsection{Properties of the Objective Function}

Property $\mathbf{1}$ The Jacobian matrix $\mathbf{J}$ of function $\mathbf{f}$ is always full rank if $\alpha>0$.

Proof If $\alpha>0, \mathbf{W}$ is a symmetric positive definite matrix. $\mathbf{R}$ is a $3 n \times 3 n$ block diagonal matrix and is the Cholesky factorization of $\mathbf{W}$. $\mathbf{L}, \mathbf{a}$, and $\mathbf{b}$ are the $\mathbf{L}^{*}, \mathrm{a}^{*}$, and $\mathrm{b}^{*}$ values for each patch in the physical scene. $\mathbf{u}$ is the $\mathrm{X}, \mathrm{Y}$, and $\mathrm{Z}$ values for each patch in the physical scene:

$\mathbf{u}=\left[x_{1}, \ldots, x_{n}, y_{1}, \ldots, y_{n}, z_{1}, \ldots, z_{n}\right]^{\top}, \mathbf{T}=\left[\begin{array}{lll}\mathbf{T}_{11} & \mathbf{T}_{12} & \mathbf{T}_{13} \\ \mathbf{T}_{21} & \mathbf{T}_{22} & \mathbf{T}_{23} \\ \mathbf{T}_{31} & \mathbf{T}_{32} & \mathbf{T}_{33}\end{array}\right]$

$\mathbf{T}$ is a $3 n \times 3 n$ matrix and $\mathbf{T}_{i j}(i, j=1,2,3)$ is a diagonal matrix with diagonal entries equal to $T_{i j}$ in the transformation matrix $T$ in Equation 2. Since $T$ is invertible, $\mathbf{T}$ is nonsingular. The Jacobian of function $\mathbf{f}$ can be written as,

$$
\mathbf{J}=\mathbf{R} \times \frac{\partial \mathbf{v}}{\partial \mathbf{u}} \times \mathbf{T} \times \mathbf{K}_{\mathbf{p}}, \frac{\partial \mathbf{v}}{\partial \mathbf{u}}=\left[\begin{array}{ccc}
0 & \frac{\partial \mathbf{L}}{\partial \mathbf{y}} & 0 \\
\frac{\partial \mathbf{a}}{\partial \mathbf{x}} & \frac{\partial \mathbf{a}}{\partial \mathbf{y}} & 0 \\
0 & \frac{\partial \mathbf{b}}{\partial \mathbf{y}} & \frac{\partial \mathbf{b}}{\partial \mathbf{z}}
\end{array}\right]
$$

Each block in $\frac{\partial \mathbf{v}}{\partial \mathbf{u}}$ is an $n \times n$ diagonal matrix. For a triplet $(L, a, b)$, its derivative with regards to $(x, y, z)$ is: 
Y. Sheng, B. Cutler, C. Chen \& J. Nasman / Perceptual Global Illumination Cancellation in Complex Projection Environments

\begin{tabular}{|c|c|c|c|c|c|c|}
\hline Methods & L-shaped & columns & green trapezoid & interior partitions & C-shaped & blue curves \\
\hline $\begin{array}{c}\text { Sheng et al. } 2010 \\
\text { (linear) }\end{array}$ & $\overline{\Delta E}=15.46$ & $\overline{\Delta E}=12.09$ & $\overline{\Delta E}=10.89$ & $\overline{\Delta E}=6.35$ & $\overline{\Delta E}=5.45$ & $\overline{\Delta E}=9.22$ \\
\cline { 2 - 7 } & $\Delta E_{m}=50.41$ & $\Delta E_{m}=49.16$ & $\Delta E_{m}=99.31$ & $\Delta E_{m}=79.23$ & $\Delta E_{m}=63.12$ & $\Delta E_{m}=84.98$ \\
\hline $\begin{array}{c}\text { Our method } \\
\text { (perceptual) }\end{array}$ & $\overline{\Delta E}=9.20$ & $\overline{\Delta E}=10.78$ & $\overline{\Delta E}=7.01$ & $\overline{\Delta E}=4.45$ & $\overline{\Delta E}=4.18$ & $\overline{\Delta E}=5.63$ \\
\cline { 2 - 7 } & $\Delta E_{m}=41.72$ & $\Delta E_{m}=40.32$ & $\Delta E_{m}=81.21$ & $\Delta E_{m}=64.20$ & $\Delta E_{m}=52.59$ & $\Delta E_{m}=71.08$ \\
\hline
\end{tabular}

Table 1: Per pixel average and maximum $\Delta E$ measurements (quantifying human perception) for the four example scenes in Figure 2 and two example scenes in Figure 4.

$$
\left[\begin{array}{ccc}
0 & \frac{116}{Y_{n}} h^{\prime}\left(\frac{y}{Y_{n}}\right) & 0 \\
\frac{500}{X_{n}} h^{\prime}\left(\frac{x}{X_{n}}\right) & -\frac{500}{Y_{n}} h^{\prime}\left(\frac{y}{Y_{n}}\right) & 0 \\
0 & \frac{200}{Y_{n}} h^{\prime}\left(\frac{y}{Y_{n}}\right) & -\frac{200}{Z_{n}} h^{\prime}\left(\frac{z}{Z_{n}}\right)
\end{array}\right]
$$

$h^{\prime}(t)$ is the derivative of function $h(t)$ in equation (3), which is always non-zero. Therefore, matrix $\frac{\partial \mathbf{v}}{\partial \mathbf{u}}$ is always nonsingular. We can draw the conclusion that $\mathbf{J}$ is always full rank because $\mathbf{R}, \mathbf{T}$, and $\mathbf{K}_{\mathbf{p}}$ are non-singular matrices.

Furthermore, $\alpha>0$ means that the matching of absolute luminance and chrominance for each patch is necessary. This property leads to the following corollary,

Corollary 1 For function $\mathbf{f}$ defined by our problem, the Gauss-Newton method will either have a valid descent direction or reach a critical point.

Property 2 The Jacobian matrix J (Eq (8)) satisfies the Lipschitz condition.

Proof We only discuss problems defined for real numbers.

A function $f: R^{N} \rightarrow R^{M}$ satisfies the Lipschitz condition if there exists a real constant $L \geq 0$, such that for any $x_{1}$ and $x_{2}$ defined in $R^{N},\left\|f\left(x_{1}\right)-f\left(x_{2}\right)\right\| \leq L\left\|x_{1}-x_{2}\right\| . f$ is also called Lipschitz continuous. Due to norm equivalence, we will focus on the proof of 2-norm.

It is easy to show that any linear mapping from $R^{N} \rightarrow R^{N}$ is bounded by the induced norm of the transformation matrix [Mrc95]. Another property of Lipschitz continuity is that if both $f$ and $g$ are Lipschitz continuous, the product $f g$ is also Lipschitz continuous. In Equation (8), $\mathbf{R}, \mathbf{T}$, and $\mathbf{K}_{\mathbf{p}}$ are all linear mappings, thus we only need to show that the operator $\frac{\partial \mathbf{v}}{\partial \mathbf{u}}$ is Lipschitz continuous. For $\mathbf{u}_{0} \in R^{3 n}, \frac{\partial \mathbf{v}}{\partial \mathbf{u}}\left(\mathbf{u}_{0}\right)$ can be decomposed into:

$\frac{\partial \mathbf{v}}{\partial \mathbf{u}}\left(\mathbf{u}_{0}\right)=\left[\begin{array}{ccc}0 & \frac{116}{\mathbf{Y}_{\mathbf{n}}} & 0 \\ \frac{500}{\mathbf{X}_{\mathbf{n}}} & -\frac{500}{\mathbf{X}_{\mathbf{n}}} & 0 \\ 0 & \frac{200}{\mathbf{Y}_{\mathbf{n}}} & -\frac{200}{\mathbf{Z}_{\mathbf{n}}}\end{array}\right] \times \frac{\partial \mathbf{h}}{\partial \mathbf{t}}\left(\left[\begin{array}{ccc}\frac{1}{\mathbf{X}_{\mathbf{n}}} & 0 & 0 \\ 0 & \frac{1}{\mathbf{Y}_{\mathbf{n}}} & 0 \\ 0 & 0 & \frac{1}{\mathbf{Z}_{\mathbf{n}}}\end{array}\right] \mathbf{u}_{0}\right)$

with $\mathbf{h}=[h(t), h(t), \ldots, h(t)]^{\top}$. Note that in Equation (10), the matrix is written in block format, each entry in the matrix represents a $n \times n$ diagonal matrix. Since the two matrices in Equation (10) are both linear mappings, we only need to prove that Jacobian $\mathbf{H}^{\prime}=\frac{\partial \mathbf{h}}{\partial \mathbf{t}}$ satisfies the Lipschitz condition. We first prove that function $h^{\prime}(t)$ is Lipschitz continuous.

$$
h^{\prime}(t)= \begin{cases}\frac{1}{3} t^{-\frac{2}{3}} & t>(6 / 29)^{3} \\ \frac{1}{3}\left(\frac{29}{6}\right)^{2} & \text { Otherwise }\end{cases}
$$

For any $t_{1}<t_{2} \in R$,

1. If $t_{1}<t_{2} \leq\left(\frac{6}{29}\right)^{3}$, then $\left|h^{\prime}\left(t_{1}\right)-h^{\prime}\left(t_{2}\right)\right|=0$, which is always bounded.

2. If $\left(\frac{6}{29}\right)^{3}<t_{1}<t_{2}$, due to the mean value theorem, there exists $t \in\left[t_{1}, t_{2}\right]$, such that,

$$
\left|h^{\prime}\left(t_{1}\right)-h^{\prime}\left(t_{2}\right)\right|=\left|h^{\prime \prime}(t)\right| \cdot\left|t_{1}-t_{2}\right| \leq \frac{2}{9}\left(\frac{29}{6}\right)^{5}\left|t_{1}-t_{2}\right| .
$$

3. If $t_{1} \leq\left(\frac{6}{29}\right)^{3}<t_{2}$, it is easy to show that

$\left|h^{\prime}\left(t_{1}\right)-h^{\prime}\left(t_{2}\right)\right| \leq \frac{2}{9}\left(\frac{29}{6}\right)^{5}\left|\left(\frac{6}{29}\right)^{3}-t_{2}\right| \leq \frac{2}{9}\left(\frac{29}{6}\right)^{5}\left|t_{1}-t_{2}\right|$

Therefore, $h^{\prime}(t)$ is Lipschitz continuous. For any two vectors $\mathbf{x}_{\mathbf{1}}, \mathbf{x}_{\mathbf{2}} \in R^{3 n}$,

$$
\left\|\mathbf{H}^{\prime}\left(\mathbf{x}_{\mathbf{1}}\right)-\mathbf{H}^{\prime}\left(\mathbf{x}_{\mathbf{2}}\right)\right\|_{2} \leq \frac{2}{9}\left(\frac{29}{6}\right)^{5}\left\|\mathbf{x}_{\mathbf{1}}-\mathbf{x}_{\mathbf{2}}\right\|_{2}
$$

Therefore, $\mathbf{H}^{\prime}$ is Lipschitz continuous, and we can safely draw the conclusion that $\mathbf{J}$ satisfies Lipschitz condition.

The second property leads to the following Corollary [LHW10].

Corollary 2 The Gauss-Newton method is locally convergent for our objective function.

\subsection{Active set Gauss-Newton algorithm}

Corollaries 1 and 2 support the use of the Gauss-Newton algorithm to achieve a fast convergence rate. Using the core idea of the active set Newton method [FJ98], we design a modified Gauss-Newton method to solve our problem. To extend the Gauss-Newton method for bound constraints, we impose the constraints on each sub-problem, which is a linear least squares problem. In each iteration, we first identify the active set, the variables that "touch" the boundary and satisfy the KKT conditions. In other words, we find all variables $x_{i}$ that satisfy the condition

$$
x_{i}=l_{i} \text { and } \nabla \phi_{i}(\mathbf{x})>0 \text {, or } x_{i}=u_{i} \text { and } \nabla \phi_{i}(\mathbf{x})<0 .
$$

These variables can be safely discarded during the solving process, a significant performance boost. Then we compute 


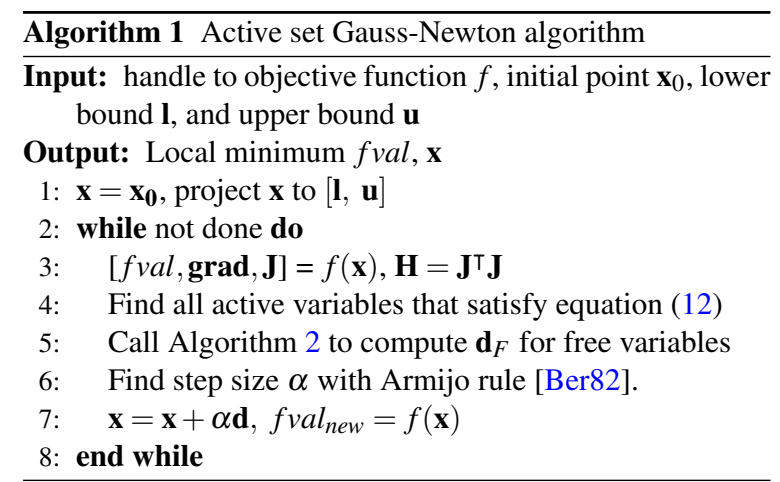

the descent direction $\mathbf{d}_{F}$ for the free variables $\mathbf{x}_{F}$ with a convex problem:

$\min \mathbf{d}_{F}^{\top}\left(\mathbf{J}^{\top} \mathbf{J}\right)_{F}+\nabla \phi_{F}(\mathbf{x}) \mathbf{d}_{F}$, s.t. $\mathbf{l}_{F}-\mathbf{x}_{F} \leq \mathbf{d}_{F} \leq \mathbf{u}_{F}-\mathbf{x}_{F}$.

For each convex sub-problem, we use a modified projected Newton method [Ber82] to achieve a fast convergence rate. Instead of diagonalizing the sub-matrix of active variables in the Hessian in the traditional projected Newton method, we directly remove the rows and columns from the Hessian for an improved running time. The detailed algorithm of our active set Gauss-Newton method is listed in algorithm 1. The modified projected Newton algorithm is listed in algorithm 2.

To guarantee the convergence of our algorithm, we perform a line search in each iteration. The proof of convergence can be made similarly to the active set Newton method [FJ98], by substituting $B_{F}^{k}$ with $\left(\mathbf{J}^{\top} \mathbf{J}\right)_{F}^{k}$. In practice, the step size computed from the line search procedure is always 1 . This is not unexpected because our objective function is Lipschitz continuous and the unconstrained GaussNewton method without line search is locally convergent. Therefore our modified algorithm can well preserve the fast convergence rates of the classical Gauss-Newton method (without line search).

\subsection{Comparison with Other Solvers}

We compare our algorithm with two general nonlinear least squares solvers, a LM method based solver implemented in $\mathrm{C}$ [Lou04] and a MATLAB solver based on the trust-region reflective method [CGT88] ("Isqnonlin" function in MATLAB). For a fair comparison, we implemented our algorithm both with MATLAB and C++. We use BLAS and LAPACK libraries from Intel MKL for both our C++ implementation and the LM solver. We ran all three algorithms for all the examples in this paper and found that our algorithm is 10 to 30 times faster than the "Isqnonlin" function, and 20 to 25 times faster than the LM solver. Figure 3 is a plot of convergence speed for each algorithm. From the curves, we see that our active set Gauss-Newton method has the fastest convergence speed. The Levenberg-Marquardt method converges

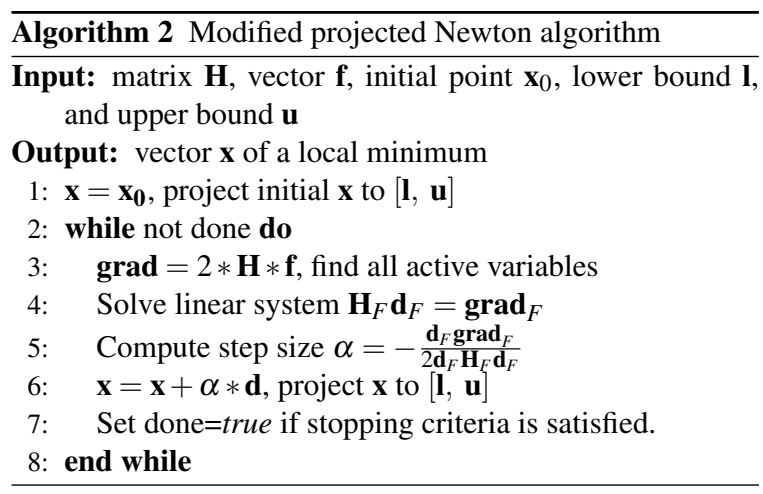

similarly fast for the first several iterations but then reverts to a slower steepest descent method.

\section{Speedup Strategies}

CUDA Implementation CUDA provides an interface to the parallel computing capability of modern GPUs. It also provides libraries for high performance numerical computing. Our implementation makes use of the CUBLAS, CUSPARSE and Thrust libraries. We also use CULA, a GPU LAPACK library, to solve linear equations on the GPU. We use single precision floating point computation with our GPU implementation. We find that the accuracy with single precision is sufficient for our application. For all the examples in this paper, the differences between the optimal result using single precision and double precision are less than $0.05 \%$ and do not cause any visual difference.

The CUDA implementation of our solver greatly improves the computing speed. On a desktop machine with an NVIDIA GeForce GTX 480 graphics card and Intel Core 2 Quad Q9450 CPU, it takes 32-50 seconds for our C++ implementation to converge for systems with $\sim 3000$ variables. The CUDA implementation requires 1.8-2.4 seconds.

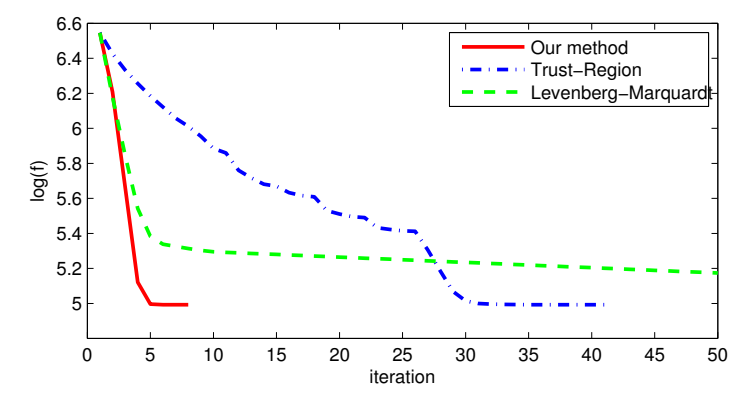

Figure 3: Comparison of the convergence speeds for different algorithms for a test case with 2880 variables. Our algorithm converges in 7 iterations, while other methods need more iterations. The log scale of this plot emphasizes the dramatic slowing of convergence with the LM method. In this example, LM needs more than 150 iterations to converge, and we do not plot all the iterations. 

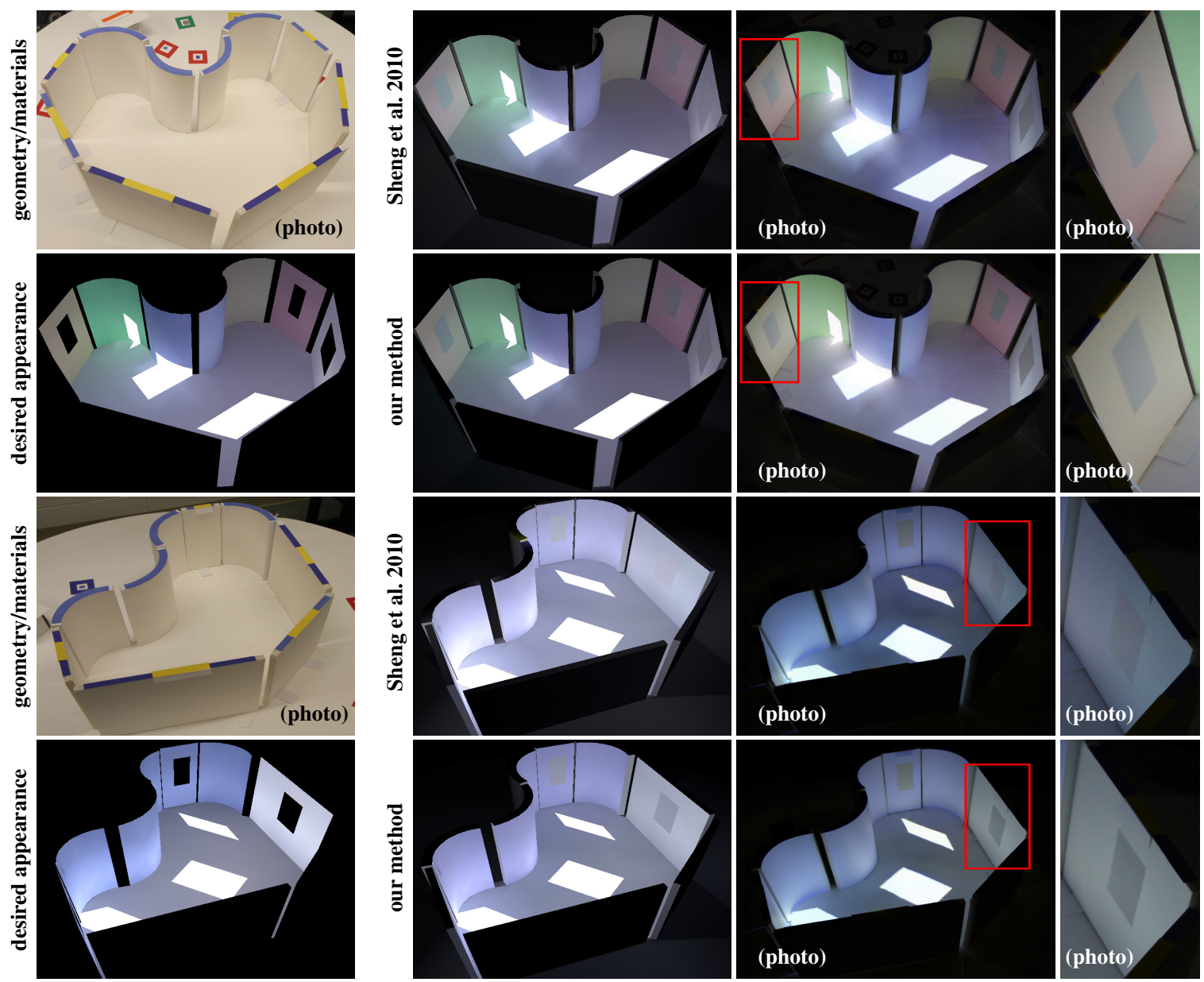

Figure 4: Two example scenes illustrate the differences between our method and Sheng et al.'s method. We present both the renderings of the expected computer simulations and photographic results from our physical projection experiments. We did not calibrate the camera; thus, comparison of viewpoint and color of the simulations to the photographs is an approximate match.

Multi-resolution A multi-resolution method has been adopted in our current implementation. We build a coarse mesh with roughly one eighth as many patches as the fine one. A simple correspondence is established between patches in different resolutions allowing the desired appearance information to be pushed from high resolution to low resolution and then to pull the solution projection data from low resolution to initialize the optimization at high resolution. Implementation details can be found in Section 5.5.2 of [She11]. In practice, with the multi-resolution strategy, the optimization algorithm is 2 to 4 times faster, converging in 0.5-1.0 seconds, which is acceptable for iterative design applications. Our implementation of the linear compensation method [SYC10] runs at $6 \mathrm{fps}, 3$ time faster than the perceptual compensation method.

\section{Physical System Demonstration}

We constructed a multi-projector environment for architectural daylighting visualization [SYYC11] to further demonstrate our perceptual global illumination cancellation algo- rithm. The projection surfaces in this space have reflectance $\rho=0.9$ and six calibrated projectors display the solution images onto these surfaces. Our perceptual cancellation algorithm preserves color fidelity and overall appearance of the desired scene significantly better than Sheng et al.'s method [SYC10]. Figure 4 shows the results of two comparison experiments both in simulation and also with photographs of our physical test environment. For the first example ("C-shaped") the apparent color of the left flat wall produced with the linear compensation algorithm is red, which does not match the desired appearance. In contrast, our perceptual method accurately portrays the desired yellow appearance of this surface. The companion video shows that the temporal appearance of the left flat wall is inconsistent when applying the linear compensation method, while our new perceptual method is consistent. In the second example in Figure 4 ("blue curves"), with our method, the sharp contrast between the blue walls and the white wall on the right is much more accurately captured. The $\Delta E$ metrics for these two examples are shown in Table 1. 


\section{Conclusions and Future Work}

We present a perceptual global illumination cancellation framework for complex multi-surface diffuse projection environments that is practical for interactive applications. We formulate the problem as a bound constrained nonlinear least squares optimization problem and design a fast solver based on active set Gauss-Newton methods. We demonstrate our algorithm within a physical multi-projector environment. Results show that our cancellation method can preserve the appearance of the desired imagery more faithfully than previous methods.

We see several interesting avenues for future work in this area. Our analysis of the result images is limited to a perpixel $\Delta E$ comparison. Future work on image-based perceptual differences [MMS04] that also take into account color differences will allow a more complete analysis of our results. Due to the limited memory on the GPU, our algorithm is currently limited to 2500 surface patches (7500 variables). Therefore, improving the scalability of the algorithm is an interesting topic. We also want to prove that our active set Gauss-Newton algorithm also has the local convergence property without using line search. The Lipschitz continuity of the Jacobian $\mathbf{J}$ and consistent performance of the technique in practice suggest that convergence is guaranteed. Finally, it may also be useful to extend the method to allow non-diffuse light transport within the system.

\section{References}

[BCNR10] Bai J., Chandraker M., Ng T.-T., RamamoorTHI R.: A dual theory of inverse and forward light transport. In European Conference on Computer Vision (2010), pp. 1-8.

[Ber82] BerTSEKAS D.: Projected newton methods for optimization problems with simple constraints. SIAM Journal on Control and Optimization 20, 2 (1982), 221-246.

[BGZ*6] Bimber O., GrundhöFer A., ZeIdLER T., DANCH D., KAPAKOS P.: Compensating indirect scattering for immersive and semi-immersive projection displays. In Proc. of the IEEE Conference on Virtual Reality (2006), pp. 151-158.

[CGT88] Conn A., Gould I., ToINT P.: Global convergence of a class of trust region algorithms for optimization with simple bounds. SIAM J. Numer. Anal. 25 (April 1988), 433-460.

[CL07] CHOU C.-H., LIU K.-C.: A fidelity metric for assessing visual quality of color images. In Proceedings of 16th International Conference on Computer Communications and Networks (Aug. 2007), pp. $1154-1159$.

[FJ98] FACCHINEI F., JÚDICE J.: An active set Newton algorithm for large-scale nonlinear programs with box constraints. SIAM J. on Optimization 8 (January 1998), 158-186.

[GB08] GRUndhöfER A., BImber O.: Real-time adaptive radiometric compensation. IEEE Transactions on Visualization and Computer Graphics 14, 1 (Jan./Feb. 2008), 97-108.

[GTGB84] Goral C., TORRance K., GreEnBerg D., BaTTAILE B.: Modelling the interaction of light between diffuse surfaces. In Computer Graphics (Proceedings of SIGGRAPH 84) (July 1984), vol. 18(3), pp. 213-222

[Lev44] LEVEnBERG K.: A method for the solution of certain non-linear problems in least squares. The Quarterly of Applied Mathematics 2 (1944), $164-168$.
[LHW10] LI C., HU N., WANG J.: Convergence behavior of Gauss-Newton's method and extensions of the smale point estimate theory. J. Complex. 26 (June 2010), 268-295.

[Lou04] LOURAKIS M.: levmar: Levenberg-Marquardt nonlinear least squares algorithms in C/C++, Jul. 2004.

[Mac42] MACADAM D.: Visual sensitivities to color differences in daylight. J. Opt. Soc. Am. 32, 5 (1942), 247-273.

[McL76] MCLAREN K.: The development of the CIE 1976 $\mathrm{L} * \mathrm{a} * \mathrm{~b} *$ uniform color-space and color-difference formula. $J$. of the Society of Dyers and Colourists 92 (1976), 338-341.

[MKO06] Mukaigawa Y., KaKinUma T., OHTA Y.: Analytical compensation of inter-reflection for pattern projection. In Proceedings of the ACM symposium on Virtual reality software and technology (2006), ACM, pp. 265-268.

[MMS04] Mantiuk R., Myszkowski K., Seidel H.-P.: Visible difference predicator for high dynamic range images. In Proceedings of IEEE International Conference on Systems, Man and Cybernetics (2004), pp. 2763-2769.

[Mrc95] MRCUN J.: Lipschitz spectrum preserving mappings on algebras of matrices. Linear Algebra and its Applications 215 (1995), $113-120$.

[MS04] Majumder A., Stevens R.: Color nonuniformity in projection-based displays: Analysis and solutions. IEEE Transactions on Visualization and Computer Graphics 10 (March 2004), 177-188.

[NPGB03] Nayar S., Peri H., Grossberg M., Belhumeur P.: A projection system with radiometric compensation for screen imperfections. In ICCV Workshop on Projector-Camera Systems (PROCAMS) (Oct 2003).

[OK10] O'Toole M., Kutulakos K. N.: Optical computing for fast light transport analysis. In ACM SIGGRAPH Asia 2010 papers (New York, NY, USA, 2010), ACM, pp. 164:1-164:12.

[RWC*98] Raskar R., Welch G., Cutts M., Lake A., STESIN L., FuCHS H.: The office of the future: A unified approach to image-based modeling and spatially immersive displays. In Proc. of SIGGRAPH 98 (July 1998), Computer Graphics Proceedings, Annual Conference Series, pp. 179-188.

[SCG*05] Sen P., Chen B., Garg G., Marschner S., Horowitz M., Levoy M., LensCh H.: Dual photography. ACM Transactions on Graphics 24, 3 (Aug. 2005), 745-755.

[She11] SHEng Y.: Interactive Daylighting Visualization in Spatially Augmented Reality Environments. PhD thesis, Rensselaer Polytechnic Institute, 2011.

[SMK05] Seitz S., Matsushita Y., Kutulakos K.: A theory of inverse light transport. In ICCV '05: Proceedings of the Tenth IEEE International Conference on Computer Vision (2005), IEEE Computer Society, pp. 1440-1447.

[SYC10] Sheng Y., YAPO T., CUTLER B.: Global illumination compensation for spatially augmented reality. In Computer Graphics Forum 29, 2 (May 2010).

[SYYC11] Sheng Y., YAPo T. C., Young C., Cutler B.: A spatially augmented reality sketching interface for architectural daylighting design. in IEEE Transactions on Visualization and Computer Graphics 17, 1 (2011), 38-50.

[WB07] Wetzstein G., Bimber O.: Radiometric compensation through inverse light transport. In Proceedings of the 15th Pacific Conference on Computer Graphics and Applications (USA, 2007), IEEE Computer Society, pp. 391-399.

[ZW97] Zhang X., WAndell B.: A spatial extension of CIELAB for digital color-image reproduction. Journal of the Society for Information Display 5, 1 (1997), 61-63. 